\title{
Dynamic Effect of Rupture of Cable in Plane Prestressed Braced Steel Moment Frame
}

\author{
Xu Jinxing ${ }^{a}$, Wang Fei ${ }^{b,{ }^{*}}$ and Li Hongming ${ }^{c}$ \\ ${ }^{1}$ School of Civil Engineering and Architecture, Jiangsu University of Science and Technology, \\ Zhenjiang, 212003, China \\ a1262843614@qq.com, 'lefm@foxmail.com, ${ }^{\mathrm{c}} 20156436 @ q q . c o m$
}

Keywords: Prestressed braced steel moment frame, Dynamic effect, Failure time, Damping ratio, Demand-capacity ratio.

\begin{abstract}
To study the dynamic effect of the prestressed braced steel moment frame caused by the sudden loss of a cable bracing, the instantaneous loading method is adopted to calculate the dynamic response of the remaining structure during and after the rupture of the cable with the simplified theoretical model as well as the finite element model. The results show that when the structure remains in the linear elastic state during the whole vibration process, the dynamic response is only related to the failure time of the broken cable and the damping ratio of the remaining structure. The maximum value of the dynamic factor approaches to 2.0. Moreover, the dynamic factor decreases when the failure time or the damping ratio increasing; but when the structure yields and enters the plastic state during the vibration, the dynamic response is also related to the demand-capacity ratio and increases with the increase of this ratio until dynamic divergence happens. The unloading impact from the broken cable is alleviated by the unbroken tensile cable, which makes the steel frame not easy to enter the plastic state.
\end{abstract}

\section{Introduction}

Recently, studying the behavior of progressive collapse of building structures have has become a hot topical. The progressive collapse [1] happens when the structure is subjected to accidental large loads, such as strong earthquake, collision, explosion, etc., which induce the initial damage or even the local failure. Then the redistribution of internal force in the structure occurs, which may cause the successive damage of other portions of the structure like a chain reaction. Eventually, the whole structure collapses. The progressive collapse of the structure often leads to heavy casualties and severe property loss. Therefore, more and more engineers and scholars have paid attention to the ability of structures to resist progressive collapse.

The prestressed braced steel moment frame (PBSMF) presents a retrofit method using steel wire strand (cable) bracings for steel moment frame. It is an innovation using flexible wire strands instead of rigid bracings. All the strands in a PBSMF have tensile force. They can increase lateral stiffness, guarantee story drift within a specified range, and reduce the residual deformation [2] as well. After the prestressed cable in a PBSMF fails suddenly, the geometric composition of the PBSMF has changed. Moreover, the storing potential energy inside it releases. The vibration of the remaining structure is induced so as to generate inertia force. At last, the system reaches a new static state of equilibrium and the redistribution of internal force is completed. That is to say, the redistribution of internal force caused by the sudden failure of the cable is a dynamic process. According to whether nonlinear and dynamic effects are considered, the analytic methods for progressive collapse can be divided into four categories: linear static analysis, nonlinear static analysis, linear dynamic analysis, and nonlinear dynamic analysis. When the static analyses are adopted, GSA [3] and DOD [4] regulate that the dynamic increase factor of loadings is 2.0. When the dynamic analyses are adopted, three methods for modeling the rupture were proposed [5]: stiffness degradation method, instantaneous loading method, and initial condition method. 
In this study, the instantaneous loading method was used to investigate the rupture process in a typical single-story PBSMF. The simplified theoretical model and the finite element model were both established to simulate the dynamic response of the remaining structure during and after the sudden loss of the cable.

\section{Single-story PBSMF for Analysis}

A typical single-story-and-single-bay plane steel moment frame with a concentrical prestressed cable X-bracing is shown in Fig. 1. Its beam is subjected to a uniformly distributed load $q$ and its weight may be neglected. Its span is 6 meters and its height is 3.6 meters. All the beam and columns have welded I-shaped section: the beam and column sections are $300 \times 300 \times 10 \times 16$ (dimensions in $\mathrm{mm}$ for depth, width, web thickness and thickness flange, respectively) and $400 \times 300 \times 10 \times 16$, respectively. The steel material used in the sections of all the members is Chinese Q235 type with elastic modulus of $2.06 \times 10^{5} \mathrm{MPa}$, and yield strength of $235 \mathrm{MPa}$. The cable with cross-sectional area of $100 \mathrm{~mm}^{2}$, elastic modulus of $1.95 \times 10^{5} \mathrm{MPa}$, breaking tensile force of $186 \mathrm{kN}$, and yield tensile force of $166 \mathrm{kN}$, is consisted of low-relaxation high strength prestressed steel wires. The failure of the right-side cable (Fig. 1) is considered here.

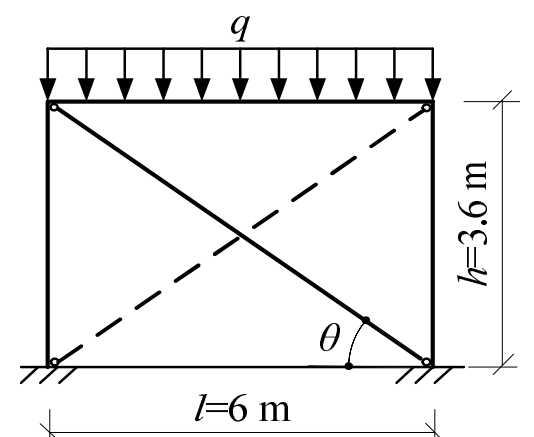

Fig. 1 Typical single-story plane PBSMF.

\section{Analyses by Using Simplified Theoretic Model}

Simplified Theoretic Model. The vertical load $q$ on the beam can be idealized as a lump of mass $m$ located at the middle of the beam, so the structure can be seen as a single-degree-of-freedom system (Fig. 2). The dotted line represents the static equilibrium position under the force on the failed beam-column joint, the magnitude of which is $p_{0}$, the same as the axial force of the right-side cable. But their directions are opposite. $u_{\mathrm{s}}$ is the corresponding lateral displacement of the lumped mass. The impact load on the remaining structure (Fig. 3) can be approximately expressed as:

$$
p(t)=\left\{\begin{aligned}
p_{0} t / t_{\mathrm{f}}, & 0 \leq t \leq t_{\mathrm{f}} \\
p_{0}, & t \geq t_{\mathrm{f}}
\end{aligned}\right.
$$

where, $t_{\mathrm{f}}$ is the failure time of the cable.

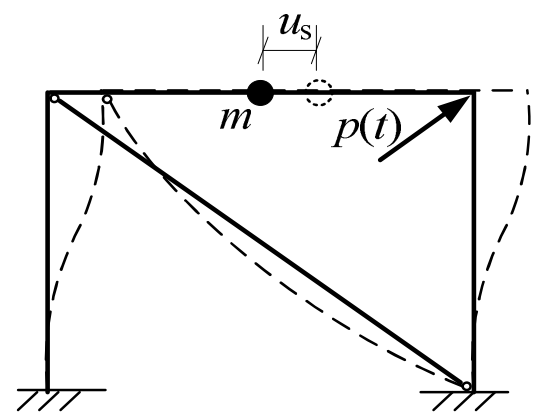

Fig. 2 Simplified frame model. 


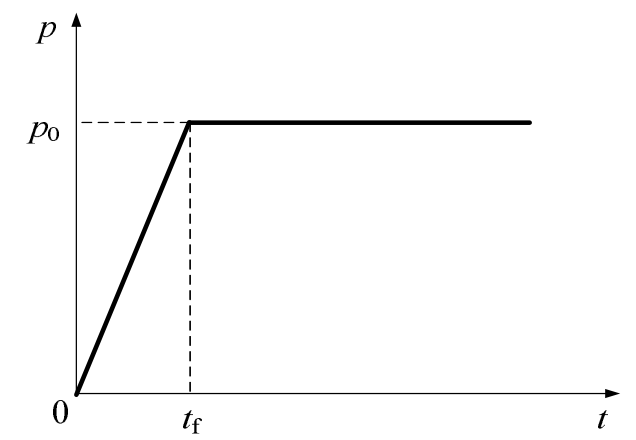

Fig. 3 Impact load when the cable fails.

Linear Elastic Analysis. In the simplified single-degree-of-freedom system with variable stiffness, the following differential equation of motion and the initial conditions are established [5]:

$$
\begin{aligned}
& \ddot{u}(t)+2 \xi \omega \dot{u}(t)+\omega^{2} u(t)=p(t) / m, \\
& u(0)=0, \dot{u}(0)=0
\end{aligned}
$$

where, $u$ is the horizontal displacement of the lumped mass, $\xi$ is the damping ratio, and the circular frequency $\omega$ is not constant.

To study the dynamic effect of the structure, the dynamic factor $R_{\mathrm{d}}$ is define as

$$
R_{\mathrm{d}}=\frac{u_{\max }}{u_{\mathrm{s}}}
$$

where, $u_{\max }$ is the peak value of lateral displacement of the lumped mass. Due to this system has two different stiffness values, for the special case $\xi=0$ or $t_{\mathrm{f}}=0$ the analytic solution of $R_{\mathrm{d}}$ can be obtained, but in a general case the numerical method should be used.

Generally, $R_{\mathrm{d}}$ depends on $\xi$ and $t_{\mathrm{f}}$ and can be expressed as a function of $\xi$ and $t_{\mathrm{f}}, R_{\mathrm{d}}\left(\xi, t_{\mathrm{f}}\right)$. It can be obtained by using the software Matlab as shown in Fig.4. At point $(0,0), R_{\mathrm{d}}$ reaches its maximum value, 2.0. But it decreases with the increase of $\xi$ or $t_{\mathrm{f}}$, and its minimum value is 1.0 , which means no dynamic effect. The influence of the damping ratio on the dynamic response is greater than that of the failure time. Fig. 5 shows that the dynamic factor $R_{\mathrm{d}}$ varies with the damping ratio as $t_{\mathrm{f}}=0$. The dynamical factor decreases with the increase of the damping ratio. When the damping ratio is 0.0 , the dynamic factor reaches a maximum value, 2.0; when the damping ratio approaches to 1.0, the dynamic factor is close to 1.0. Fig. 6 shows how the dynamic factor varies with the failure time as $\xi=0$. The decay curve of the dynamic factor has a wave shape. When the failure time is 0.0 , the dynamic factor reaches the maximum value, 2.0; when the failure time is greater than the base period of the remaining structure, the dynamic factor is less than 1.2 and approaches to 1.0.

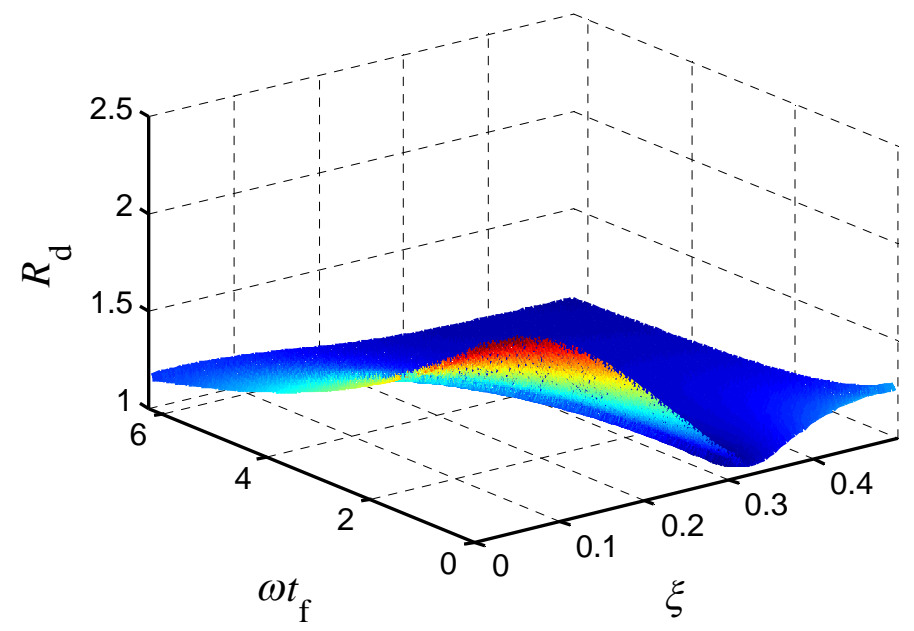

Fig. 4 Dynamic factor of lateral displacement. 


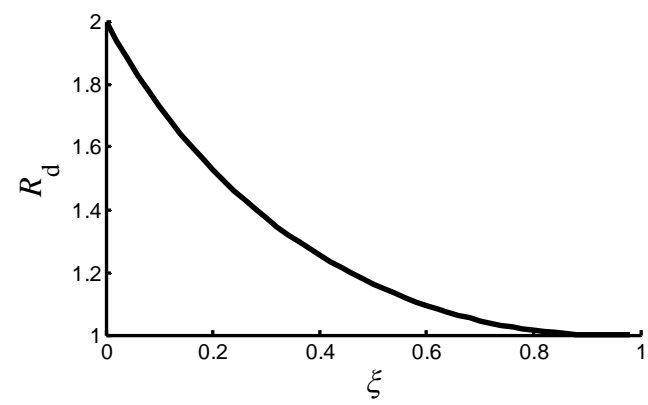

Fig. 5 Dynamic factor varies with damping ratio when $t_{\mathrm{f}}=0$.

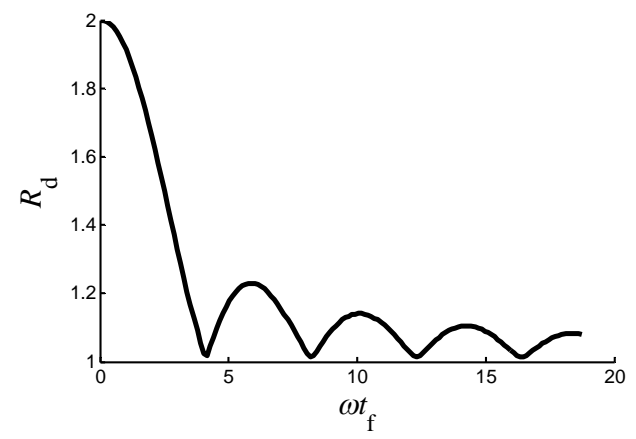

Fig. 6 Dynamic factor varies with failure time when $\xi=0$.

The rupture time of the cable is very short, and it can be taken as 0.1 times the period of the remaining structure [4]. For steel frame structures, the damping ratio is 0.02 . Therefore, the dynamic factor is 1.93 . If the damping ratio increases to 0.05 , the dynamic factor reduces to 1.84 . If the rupture time is taken as 0.02 times the period, the dynamic factor is 2.00 when the damping ratio is 0.02 ; the dynamic factor becomes 1.73 when the damping ratio rises to 0.05 .

Elastic-plastic Analysis.

Definition of Demand-capacity Ratio. To indicate the relative magnitude of the impact load on the structure, the demand-capacity ratio DCR is defined as

$$
\mathrm{DCR}=\frac{p_{0}}{p_{\mathrm{y}}}
$$

where, $p_{\mathrm{y}}$ is the tensile force of the cable when the frame yields.

To indicate the plastic deformation extent, the ductility factor $\mu$ is defined as

$$
\mu=\frac{u_{\max }}{u_{\mathrm{y}}}
$$

where, $u_{\mathrm{y}}$ represents the yield displacement. When $\mu<1$, the single-degree-of-freedom system is in the elastic state; when $\mu \geq 1$, the system is in the elastic-plastic state.

When $\mathrm{DCR} \leq 1$, which means $p_{0} \leq p_{\mathrm{y}}$, the relationships between and is expressed as

$$
\mathrm{DCR}=\frac{p_{0}}{p_{\mathrm{y}}}=\frac{u_{\mathrm{s}}}{(1+k) u_{\mathrm{y}}}
$$

where, $k$ is the ratio of the lateral stiffness of the unbroken cable bracing to the stiffness of the frame without bracings. The following function can be derived from Eqs. (3), (5), and (6):

$$
\mu=\frac{\mathrm{DCR} \cdot R_{\mathrm{d}}}{1+k}
$$

So the ductility factor is influenced by $k$ as well as DCR and $R_{\mathrm{d}}$. When $k$ increases, $\mu$ decreases. 
Parameters for Elastic-plastic Analysis. To establish the single-degree-of-freedom model for elastic-plastic analysis, an elastic-perfectly plastic restoring force model is used. $p_{\mathrm{y}}$ can be obtained by the limit state analysis and written as:

$$
p_{\mathrm{y}}=\frac{4 f_{\mathrm{y}} W_{\mathrm{p}}}{h \cos \theta}
$$

where, $W_{\mathrm{p}}$ is the plastic flexural modulus of the cross section of the columns and $f_{\mathrm{y}}$ is the yield strength of the steel. Table 1 shows the parameters used for the elastic-plastic time-history analysis. $t$ and $\Delta t$ represent the total time and time step of the analysis, respectively. The Newmark- $\beta$ method is used in the nonlinear time-history analysis.

Table 1. Parameters for elastic-plastic time-history analysis.

\begin{tabular}{ccccccc}
\hline$m / \mathrm{kg}$ & $p_{\mathrm{y}} / \mathrm{kN}$ & $u_{\mathrm{y}} / \mathrm{mm}$ & $t_{\mathrm{f}} / \mathrm{ms}$ & $t / \mathrm{s}$ & $\Delta t / \mathrm{ms}$ & $\xi$ \\
\hline $2.16 \times 10^{4}$ & 223 & 31 & 37 & 12 & 5 & 0.02 \\
\hline
\end{tabular}

Results of Nonlinear Time-history Analysis. Table 2 lists the values of $R_{\mathrm{d}}, \mu$ and $t_{\mathrm{max}}$, the time when the peak value of the frame drift occurs. The time-history curves of the frame drift for different DCRs are shown in Fig. 7. If DCR $<1.30$, the system is in the elastic state. The value of $R_{\mathrm{d}}$ is 1.93 and remains the same. The occurring time of the peak drift is $145 \mathrm{~ms}$. But if $\mathrm{DCR} \geq 1.30$, the system is in the elastic-plastic state. $R_{\mathrm{d}}$ increases and the occurring time of the peak drift delays when DCR is increasing. However, the failure force of the cable is less than the value of $p_{\text {y }}$ given in Table 1 , the system will not enter the plastic state before the rupture happens.

Table 2. Dynamic factor, ductility factor and occurring time of peak drift.

\begin{tabular}{cccccccccc}
\hline DCR & 0.10 & 0.30 & 0.50 & 0.70 & 0.90 & 1.10 & 1.30 & 1.50 & 1.70 \\
\hline$R_{\mathrm{d}}$ & 1.93 & 1.93 & 1.93 & 1.93 & 1.93 & 1.93 & 1.93 & 2.03 & 2.29 \\
$\mu$ & 0.08 & 0.25 & 0.41 & 0.57 & 0.74 & 0.90 & 1.07 & 1.29 & 1.65 \\
$t_{\max } / \mathrm{ms}$ & 145 & 145 & 145 & 145 & 145 & 145 & 145 & 160 & 185 \\
\hline
\end{tabular}

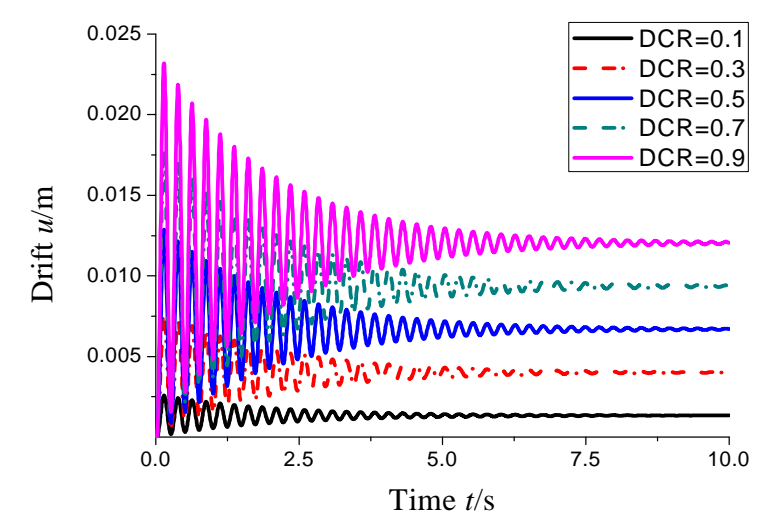

(a) DCR: $0.1,0.3,0.5,0.7$ and 0.9

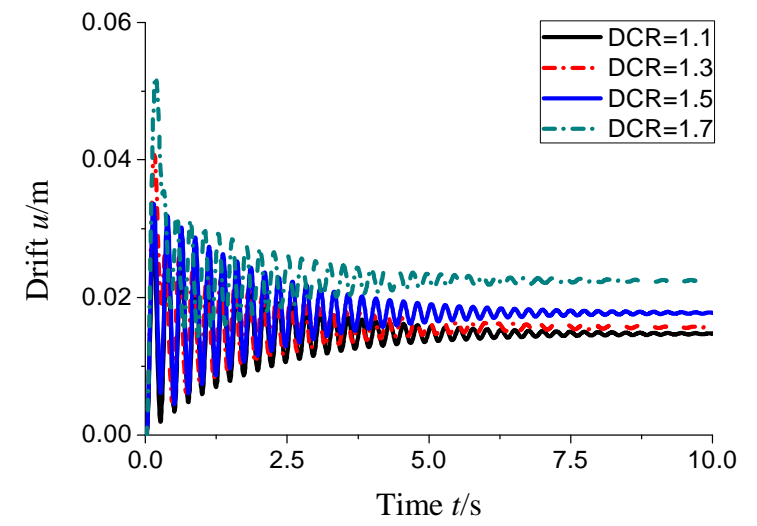

(b) DCR: 1.1, 1.3, 1.5 and 1.7

Fig. 7 Time-history curves of frame drift for different DCRs by using simplified model.

\section{Analyses by Using Finite Element Model}

Analysis Method. Using the instantaneous loading method, the time-history analysis should be carried out in two steps: (1)remove the failed cable and apply its axial force reversely on the remaining structure (Fig. 8a); (2)then apply the impact load, as shown in Fig. 3, at the failed joint (Fig. $8 b)$. Its direction is same as the axial force of the failed cable. 


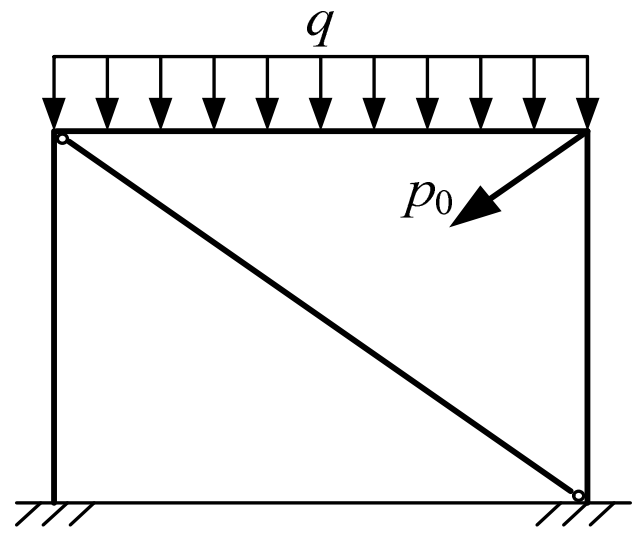

(a) Step 1

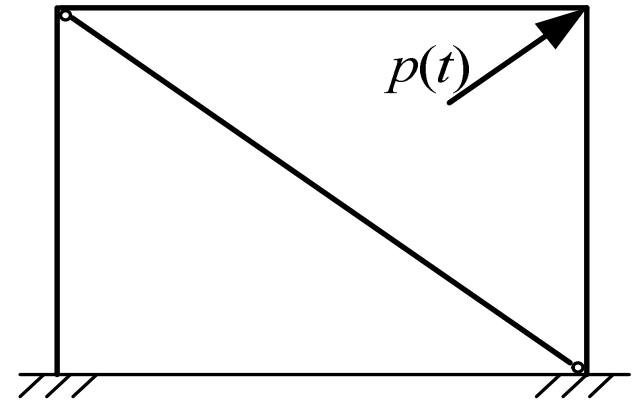

(b) Step 2

Fig. 8 Steps of instantaneous loading method.

The pushover analysis of the remaining structure was conducted by using the software SAP2000. The frame element with concentrated plastic hinges was adopted. The M3 hinges were assigned at both the ends and the middle part of the beam. The PMM hinges were assigned at the two ends of the column. The pushing force with the same direction as the impact load was applied to the failed joint (Fig. 8). SAP2000 was also used for the nonlinear time-history analysis and the location of plastic hinges is the same as that in the pushover analysis.

Parameters for Time-history Analysis. The definition of the dynamic factor is the same as Eq. (3). The definition of DCR is similar to Eq. (4), in which $p_{\mathrm{y}}$ should be replaced as the ultimate load $p_{\mathrm{u}}$ with value of $234 \mathrm{kN}$ obtained through the pushover curve. It is larger than the breaking force of the cable.

The modal analysis for the remaining structure after the failure of the right-side cable is carried out to acquire the period and the first- and second-order frequencies. The former is used to determine the failure time $t_{\mathrm{f}}$ and the later are used to determine the Rayleigh damping factors. The failure time $t_{\mathrm{f}}$ is taken as 0.1 times the base period of the remaining structure and the modal damping ratio is 0.02 . The main parameters are shown in Table 3, in which $\alpha, \beta$ are the mass damping factor and the stiffness damping factor, respectively.

Table 3. Parameters for finite element analysis.

\begin{tabular}{ccccc}
\hline$t_{\mathrm{f}} / \mathrm{ms}$ & $\alpha$ & $\beta$ & $t / \mathrm{s}$ & $\Delta t / \mathrm{ms}$ \\
\hline 34 & 0.6943 & $1.427 \times 10^{-4}$ & 12 & 5 \\
\hline
\end{tabular}

Results of Time-history Analysis. Table 4 lists the values of $R_{\mathrm{d}}, \mu$ and $t_{\max }$. The time-history curves of the frame drift for different DCRs are shown in Fig. 9. If DCR $<0.4$, the structure is in elastic state. $R_{\mathrm{d}}$ remains constant basically and is close to the result of the simplified single-degree-of-freedom model. If $\mathrm{DCR} \geq 0.4$, the steel frame is still in the elastic state but the unbroken left-side cable has shown the plastic behavior. $R_{\mathrm{d}}$ decreases slightly when DCR increases. Therefore, $u$ decreases slightly compared with the results of the single-degree-of-freedom model. Since the frame is always in the elastic state, the occurring times of the peak drift value for different DCRs are all 204ms.

Table 4. Dynamic factor, ductility factor and occurring time of peak drift.

\begin{tabular}{cccccccc}
\hline DCR & 0.10 & 0.20 & 0.30 & 0.40 & 0.50 & 0.60 & 0.70 \\
\hline$R_{\mathrm{d}}$ & 1.94 & 1.94 & 1.93 & 1.90 & 1.88 & 1.88 & 1.87 \\
$\mu$ & 0.08 & 0.16 & 0.25 & 0.32 & 0.40 & 0.48 & 0.56 \\
$t_{\max } / \mathrm{ms}$ & 204 & 204 & 204 & 204 & 204 & 204 & 204 \\
\hline
\end{tabular}




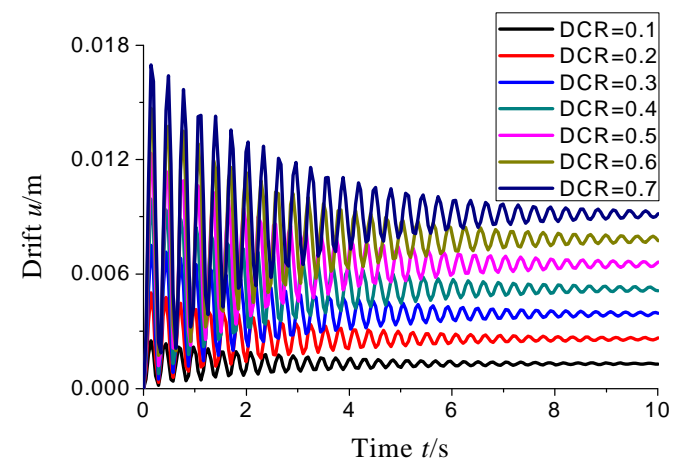

Fig. 9 Time-history curves of frame drift for different DCRs by using finite element model.

\section{Conclusions}

To study the dynamic effect of the prestressed braced steel moment frame caused by the sudden loss of the cable, the dynamic response of the remaining structure is investigated by using both the simplified single-degree-of-freedom model and the finite element model, and the main conclusions are as follows:

(1) When the structure is in the linear elastic state, the dynamic effect is only related to the failure time of the cable and the damping ratio and is not related to the tensile force of the cables. The maximum value of the dynamic factor is close to 2.0 and it decreases with the increase of the failure time and the damping ratio.

(2) When the structure is in the elastic-plastic state, the dynamic effect is related to not only the failure time and the damping ratio, but also the tensile force of the cables. The dynamic factor increases with the increase of the demand-capacity ratio until dynamic divergence appears.

(3) The tensile force of the unbroken cable alleviates the impact from the broken cable and leads to the steel frame postponed to enter the plastic deformation.

\section{Acknowledgement}

The authors would like to deeply appreciate the support of the Natural Science Fund Project of Colleges in Jiangsu Province (No. 15KJB560004).

\section{References}

[1] X. Z. Zhao, S. Yan and Y. Y. Chen, Research method and current situation of progressive collapse of large span spatial structures, J. Architectural Structure, 34(4) (2013) 1-14.

[2] A. L. Zhang, L. Zhao and X. Liu, Study on wire rope brace design method of prestressed braced steel moment frame, Open Civil Eng. J. 9(1) (2015) 21-31.

[3] GSA2013. General services administration alternate path analysis and design guidelines for progressive collapse resistance, Washington: U.S. General Services Administration, 2013.

[4] DoD2010. Design of buildings to resist progressive collapse, Washington: U.S. Department of Defense, 2010.

[5] N. Buscemi and S. Marjanishvili, SDOF model for progressive collapse analysis, Structures Congress 2005, ASCE. New York, 2005. 